\title{
Identification of grapevine rootstock cultivars using expressed sequence tag-simple sequence repeats
}

\author{
X.C. Fan ${ }^{1 *}$, J.Q. Chu ${ }^{2,3 *}$, C.H. Liu ${ }^{1}$, X. Sun ${ }^{2,3}$ and J.G. Fang ${ }^{2,3}$ \\ ${ }^{1}$ Zhengzhou Fruit Research Institute, Chinese Academy of Agriculture Science, \\ Zhengzhou China \\ ${ }^{2}$ College of Horticulture, Nanjing Agricultural University, Nanjing, China \\ ${ }^{3}$ Jiangsu Fruit Crop Genetics Improvement and Seedling Propagation \\ Engineering Center, Nanjing, China \\ *These authors contributed equally to this study. \\ Corresponding author: X.C. Fan \\ E-mail: xiucfan@163.com
}

Genet. Mol. Res. 13 (3): 7649-7657 (2014)

Received March 9, 2013

Accepted July 8, 2014

Published September 26, 2014

DOI http://dx.doi.org/10.4238/2014.September.26.2

\begin{abstract}
Grapevine (Vitis) rootstock varieties or cultivars are used to confer resistance and tolerance to insect and disease pests, unfavorable soil conditions, and other environmental conditions to cultivars that are susceptible to these conditions but otherwise have desired properties. The need to genotype and thoroughly identify grapevine rootstock varieties in the grape industry has become increasingly critical as more and more varieties are bred or selected. Although DNA markers have advantageous applications in plant identification, markers developed from classic DNA fingerprint analysis methods are not practical for plant cultivar identification. The manual cultivar identification diagram (MCID), which was previously developed in our research group, has been shown to select DNA markers that are relatively more exploitable in identifications of genotyped plant individuals. Using this MCID strategy and expressed sequence tag-simple sequence repeat (EST-
\end{abstract}


SSR) markers, we identified 22 grapevine rootstock cultivars of diverse origin. All cultivars were clearly separated by fingerprints of seven pairs of EST-SSR primers and the grapevine rootstock CID (V-R-CID) generated is both practical and referable for the identification of any grapevine rootstock cultivars studied here. Furthermore, fewer primers can be used to distinguish all cultivars using this approach since the fingerprint from each primer pair could be used several times once it is generated. This initial version of V-R-CID can be made more informative with the identification and incorporation of more cultivars, thus providing better service to the grape industry.

Key words: Grape; Rootstock; Cultivar identification diagram; EST-SSR

\section{INTRODUCTION}

Grapevine is a socially and economically important crop in many countries worldwide. Rootstocks derived from hybridizing wild American Vitis species (predominantly Vitis riparia) with root resistance to pests, disease, soil, and water stresses have been successfully used to rescue the European viticulture industry. With the development of the global grape industry, the use of grafted grape seedlings and the grafting of plants established are becoming increasingly popular, which in turn has focused increased attention on research related to breeding grapevine rootstocks. With more grapevine rootstock cultivars being bred, accurate and rapid identification of these cultivars becomes increasingly necessary for both breeders and commercial companies. Only a few studies have been performed on the use of molecular markers for the identification of grapevine rootstock cultivars (Lopes et al., 1999; Fossati et al., 2001). Although phylogenetic tree-based dendrograms that were generated from cluster analysis of DNA banding patterns of cultivars have indicated the levels of genetic diversity in individual plants, they are not suitable for the simple and practical identification of rootstock cultivars needed by the grapevine industry. Therefore, there is a growing need to make identification of grapevine rootstock cultivars reliable, easy, and referable, as it is vital for the nursery industry and to growers for purposes of protecting plant patents and for providing genetically uniform plants.

Molecular markers are advantageous in that they are not affected by the environment and can provide a powerful tool for the proper characterization of cultivars. Various DNAbased markers have been developed and used in studies on genetic diversity, fingerprinting, and origins of cultivars in different fruits (Fang et al., 2005; Cheng et al., 2009; D'Onofrio et al., 2009; Elidemir et al., 2009; Melgarejo et al., 2009; Papp et al., 2010). Among the several markers available, simple sequence repeat (SSR) markers are widely preferred for genotype characterization, genome analysis, and gene mapping in various species, as these are polymerase chain reaction (PCR)-based, co-dominant, locus-specific, highly reproducible, hypervariable, informative, and reasonably easy to use (Powell et al., 1996). Applications of SSR markers in the identification of grapevine cultivars are of great interest for grape production and research. The objective of this study was to employ the manual cultivar identification diagram (MCID) strategy for practical, efficient, recordable, and referable identification of 
grapevine rootstock cultivars by creating a cultivar identification diagram of the 22 selected grapevine rootstock cultivars (V-R-CID). The V-R-CID can be readily employed in cultivar identification with advantages of high referability and ease of use, workability, and flexibility through the addition of new cultivars as their data become available. In addition, the use of this MCID strategy will provide valuable information and a theoretical basis for the identification of cultivars, genetic diversity analysis, and genetic improvement of crops at the molecular level, besides being an essential requirement in granting protection to new varieties through distinctness, uniformity, and stability (DUS) testing (Lu et al., 2009).

\section{MATERIAL AND METHODS}

\section{Plant materials and genomic DNA extraction}

A total of 22 grapevine rootstock genotypes (Table 1) were used in this study. Total genomic DNA of each genotype was extracted from young leaves using the modified cetyltrimethylammonium bromide (CTAB) method (Murray and Thompson, 1980; Bousquet et al., 1990). The extracted DNA was diluted to a final concentration of $30 \mathrm{ng} / \mu \mathrm{L}$ with $1 \mathrm{X}$ TE buffer and stored at $-20^{\circ} \mathrm{C}$.

\begin{tabular}{|c|c|c|c|c|c|}
\hline No. & Cultivar & Origin & No. & Cultivar & Origin \\
\hline 1 & Pulliat & * & 12 & Youmute & * \\
\hline 2 & $420 \mathrm{~A}$ & France & 13 & Eldorado & * \\
\hline 3 & Champinii & USA & 14 & Grand glaber & * \\
\hline 4 & $101-14$ & France & 15 & Hybrid Franc & France \\
\hline 5 & Mcadamsu & Hungary & 16 & Rupestris du Lot & France \\
\hline 6 & Palette50 & France & 17 & shen528 & $*$ \\
\hline 7 & $520 \mathrm{~A}$ & USA & 18 & Rupestris & USA \\
\hline 8 & $5 \mathrm{C}$ & Hungary & 19 & shen 530 & $*$ \\
\hline 9 & SO4 & Germany & 20 & Dogridge & USA \\
\hline 10 & Golire & France & 21 & Saltcreek & USA \\
\hline 11 & Freedom & USA & 22 & 1103 & Italy \\
\hline
\end{tabular}

*Unknown origin of the cultivar.

\section{Amplification of EST-SSR markers}

A $20-\mu \mathrm{L}$ reaction mixture containing $10.5 \mu \mathrm{LddH}_{2} \mathrm{O}, 2 \mu \mathrm{L} 10 \mathrm{X}$ buffer, $1.2 \mu \mathrm{L} 25 \mathrm{mM}$ $\mathrm{MgCl}_{2} 1.6 \mu \mathrm{L} 2.5 \mathrm{mM}$ dNTPs, $0.8 \mu \mathrm{L} 10 \mathrm{pmol} / \mu \mathrm{L}$ primer, $3 \mu \mathrm{L} 30 \mathrm{ng} / \mu \mathrm{L}$ genomic DNA, and $0.1 \mu \mathrm{L} 5 \mathrm{U} / \mu \mathrm{L}$ DNA polymerase was prepared. PCR was carried out in an Autorisierter Thermocycler (Eppendorf, Hamburg, Germany), which was programmed as follows: pre-denaturation for $5 \mathrm{~min}$ at $94^{\circ} \mathrm{C}$; 35 cycles each of denaturation for $45 \mathrm{~s}$ at $94^{\circ} \mathrm{C}$, an annealing step for $40 \mathrm{~s}$ at the corresponding annealing temperatures (Table 2), and an extension step for $1 \mathrm{~min}$ at $72^{\circ} \mathrm{C}$; and a final extension step of $10 \mathrm{~min}$ at $72^{\circ} \mathrm{C}$.

\section{EST-SSR analysis}

From a set of EST-SSR primers, reproducible polymorphic bands were developed with 
seven pairs of randomly selected primers (Table 2). The PCR products were electrophoresed on $6 \%$ acrylamide gels (19:1 acrylamide-bisacrylamide, $7.5 \mathrm{M}$ urea) in $1 \mathrm{X}$ TBE buffer (50 mM Tris, $50 \mathrm{mM}$ boric acid, $1 \mathrm{mM}$ EDTA, pH 8.0 ) at $200 \mathrm{~V}$ and $100 \mathrm{~mA}$ for $1 \mathrm{~h}$, depending on the fragment sizes. Each gel included lanes of the molecular size marker. Gels were silver stained after electrophoresis, according to Bassam's method (Bassam, 1991). In order to obtain reproducible, accurate, and clear banding patterns, all amplifications were repeated at least three times each.

\section{Data analysis}

Only clear and unambiguous bands in the photographic prints of gels were manually chosen and scored for each cultivar by each primer. When a cultivar had a specific band in the fingerprint generated from one primer, it could be individually separated out, while cultivars sharing the same banding pattern were separated into the same sub-group, and the remaining cultivars were separated into another sub-group. On this basis, all the grapevine rootstock cultivars were gradually and completely separated from each other as an increasing number of primers were employed.

\begin{tabular}{llc} 
Table2. Seven pairs EST-SSR primers in this study. \\
\hline Primer & Nucleotide sequence $\left(5^{\prime} \rightarrow 3^{\prime}\right)$ & Annealing temperature $\left({ }^{\circ} \mathrm{C}\right)$ \\
\hline E10 & ACCGCTTCTTTGCCTCTTCT & 55.0 \\
E14 & GATAAACCCCTCCAGCAAT & 58.0 \\
E30 & ACCACTGTGTCCTCCACTCC & 57.6 \\
E45 & CATGAAAAGCATGCAGCAAT & 58.4 \\
E69 & GACCATGTTCTCTCCGCTTC & 58.6 \\
E70 & GAGATGTACTCGTCCTCCAT & 58.6 \\
E77 & CACAGCCGCTCCTCACTACACTCTCTCC & 57.8 \\
& AAGCCGATCCCATAGTCCT & 5 \\
\hline
\end{tabular}

\section{Test of workability of the cultivar identification diagram}

In order to check the workability of the V-R-CID and demonstrate its applicability, several grapevine rootstock cultivars were randomly chosen from the inter- and intra-groups of the V-R-CID, and were used for verification. The corresponding primers used for the separation of each group were easily picked out from the diagram.

\section{RESULTS}

\section{Cultivar identification}

To determine the suitability of the EST-SSR marker technique in identifying the grapevine rootstock cultivars, a set of 103 pairs of EST-SSR primers were available for screening and the annealing temperature for each primer pair was screened based on the quality and 
reproducibility of its banding patterns. Seven pairs of primers were successfully screened and all 22 grapevine rootstock cultivars could be thoroughly identified by the joint use of these seven primer pairs (Table 2). A notable example of the EST-SSR pattern, obtained with primer E45, is shown in Figure 1A. The EST-SSR primer E45 was the first one screened out, and was used to amplify the 22 grapevine rootstock cultivars. Electrophoresis results showed that one grapevine rootstock cultivar, coded as 19 , generated a uniform, clear, and reproducible 220bp band, which was absent in all of the other cultivars (Figure 1A). When this special band was selected for cultivar identification, the cultivar coded as 19 could be directly identified. Similarly, two other grapevine rootstock cultivars, coded as 16 and 17, could be picked out from the pool of cultivars based on the specific 180-bp band (Figure 1A). The remaining 19 grapevine rootstock cultivars could be separated into three groups by two bands of $160 \mathrm{bp}$ and $200 \mathrm{bp}$, respectively. Twelve rootstock cultivars generated two bands of $160 \mathrm{bp}$ and 200 bp (Figure 1A), of which four generated only 160-bp bands, and two generated only 200-bp bands. Eventually, the 22 grapevine rootstock cultivars were successfully identified into five groups with the use of the primer E45 (Figure 1A). Primer E69 was chosen to differentiate four of these five groups of grapevine rootstock cultivars. Because the cultivar coded 19 was in its own group, it was not subjected to any further testing. Among the four remaining groups, polymorphic bands were only observed in the second group. Since the other three groups could not be differentiated using primer E69, primers E70 (Figure 1B) and E14 were chosen to further distinguish cultivars within these three groups. Ultimately, the four groups of cultivars could be successfully separated into several secondary groups with the use of primers E69, E70, and E14. A further example of such separation is the EST-SSR pattern obtained with primer E10, which had several polymorphic bands, indicating that the cultivars in this group could be successfully differentiated from each other (Figure 1C). After following this strategy, the original 22 grapevine rootstock cultivars were completely differentiated from each other using all seven primer pairs (Figure 2), and the grapevine rootstock cultivar identification diagram (V-R-CID) was constructed for which the size of polymorphic bands used was marked at the corresponding separation steps. All of the primers and polymorphic bands shown on the V-RCID provide relevant information for use in the identification of cultivars as needed, making this CID practically workable and referable in the grape industry.

\section{Test of utilization and workability of the diagram in cultivar identification}

An important aim of this study was to learn how to use the EST-SSR markers to distinguish the 22 grapevine rootstock cultivars. In addition, we endeavored to generate a referable V-R-CID for identification of cultivars in future nursery industry practice and cultivarright protection. This second aim was an interesting, newly inventive, and more important purpose, which called for verification of the utilization, workability, and efficiency of the diagram in cultivar identification. To achieve this, 10 grapevine rootstocks, namely, 'Pulliat', '420A', 'Mcadams', 'Freedom', 'Hybrid Franc', 'Rupestris du Lot', 'shen528', 'shen530', 'Dogridge', and 'Saltcreek', were selected randomly from the inter- and intra-groups or subgroups in the CID and used to verify the scientific aspects of this method. Based on the location of these cultivars in the CID, it was easy to find the primer needed to separate them. For these 10 cultivars, the primers E14, E45, E70, and E77 (Figure 2) were used. The PCR results clearly showed that the 10 grapevine rootstock cultivars could be identified with five specific 
bands as anticipated in Figure 2. For example, primer E45 was used to amplify the genomic sequences of 'shen530' and 'Dogridge' (Figure 3), where a specific band, whose fragment size was approximately $220 \mathrm{bp}$, was used for cultivar identification. Primer E70 was used to amplify the genomic sequences of 'Pulliat' and 'Mcadams', or 'Rupestris du Lot' and 'shen528' (Figure 3), where the specific bands, whose fragment sizes were approximately $230 \mathrm{bp}$ and $240 \mathrm{bp}$ respectively, were used for cultivar identification. Similarly, to separate ' $420 \mathrm{~A}$ ' and 'Hybrid Franc', or 'Freedom' and 'Saltcreek', primers E77 and E14 were needed, respectively. This verification test confirmed that this method is both accurate and reliable.

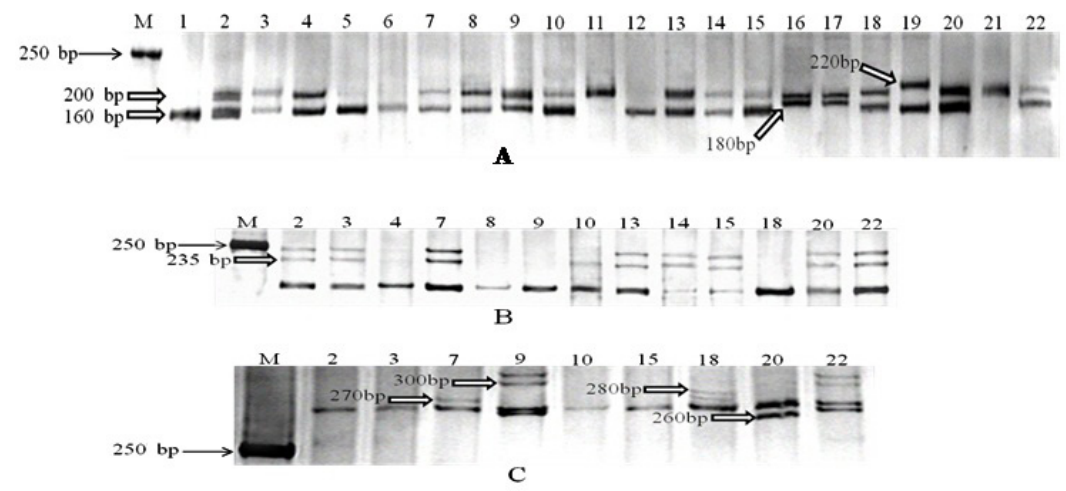

Figure 1A. EST-SSR marker patterns of 22 grapevine rootstock genotypes obtained with primer E45. Horizontal arrows indicate the specific bands. The lane numbers correspond to the cultivar codes in Table 1. Lane $M=$ DNA size marker. B. and C. ESR-SSR profiles obtained with EST-SSR primers. Horizontal arrows indicate the specific bands. The lane numbers correspond to the code in Table 1. Lane $M=$ DNA size marker. B obtained with the primer E69, C obtained with the primer E10.

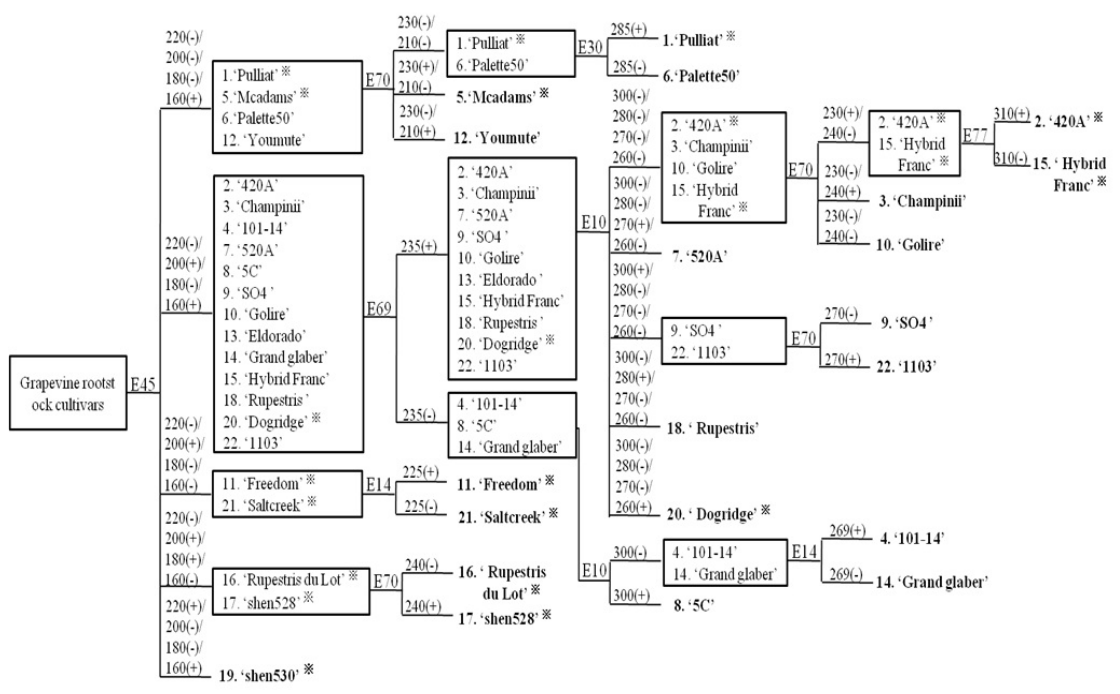

Figure. 2 Classification of 22 grapevine rootstock cultivars based on seven ESR-SSR primers. The number above each horizontal line in the Figure means the size of the band in bp. " + " = band present; "“-" = band absent. ' $※$ ' = the cultivar was used for validation. The bolded names of the cultivars mean this cultivar was separated out at this point. 


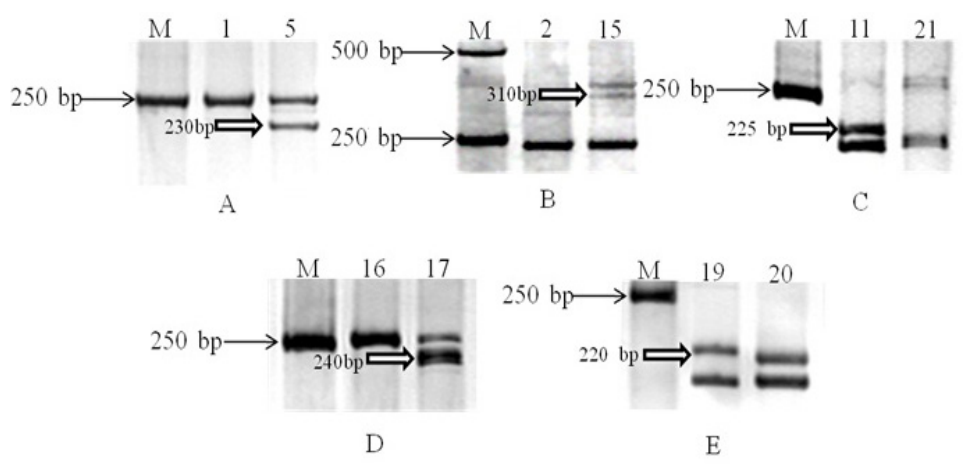

Figure. 3 ESR-SSR profiles obtained with ESR-SSR primers. Horizontal arrows indicate the specific bands. The lane numbers correspond to the code in Table 1. Lane $M=$ DNA size marker. A. and D. obtained with the E70 primer, B. obtained with the E77 primer, C. obtained with the E14 primer, E. obtained with the E45 primer.

\section{DISCUSSION}

DNA-based molecular markers have served as versatile tools in various fields including taxonomy, genetic engineering, marker-assisted selection (MAS), cultivar identification, and variability studies. These classes of markers are found in abundance and are relatively more precise, thus providing an opportunity for direct comparison of genetic materials. Furthermore, they are not affected by different environmental conditions or by the developmental stage of plants (Reddy et al., 2002). Despite these advantages, DNA markers have not been easily used in genotyping plants, and the situation appears to be more serious than anticipated. The question of whether DNA markers can be readily used in the identification of plant varieties often yields a negative response from many scientists. No efficient approaches have been developed to use DNA markers easily and efficiently in plant cultivar identification apart from the use of phylogenetic clusters or some fingerprints. Apparently, the clusters formed in phylogenetic trees do not provide the referable information needed for the identification of plant samples, while fingerprinting cannot present all of the fingerprints of many cultivars simultaneously for easy identification. These weaknesses could likely be attributed to the fact that no analysis has yet been capable of connecting the information of DNA fingerprints with that of cultivars in an easy, clear, and readable way. The new approach developed and used in this study can employ DNA markers efficiently to distinguish the cultivars as desired. It has the advantages of low cost, timeliness, and objectivity among others. This strategy can harness the power of DNA markers in plant cultivar identification and can utilize the polymorphic bands of each primer to gradually distinguish species, cultivars, and individual plants. In the case of cultivars, a CID can ultimately be constructed for further use.

Botanical classification of species and cultivars used as grapevine rootstocks is somewhat controversial, partly because of the ease of inter-specific hybridization, which creates numerous intermediate types, and blurs species boundaries. Therefore, these factors make it difficult to avoid situations of homonyms or synonyms within the materials, and highlight the need to identify grapevine rootstock species and cultivars for conservation studies and for the optimal use of germplasm resources as well as plant variety protection. The ability to distinguish cultivars could be greatly enhanced by using appropriate molecular markers (Iez- 
zoni and Brettin, 1998). The EST-SSR marker technique was selected in this study due to its efficiency and ease of use. The most important aim of this study was, however, not limited to knowing how to use EST-SSR markers to distinguish the 22 grapevine rootstock cultivars, which focuses on the utilization of DNA fingerprints in identifying plant cultivars, but this study also sought to develop a new strategy for the proper utilization of DNA markers in the separation of grapevine rootstock cultivars. This methodology could also be adopted as a universal strategy for use in distinguishing cultivars and seed samples of other plant species.

By deployment of the MCID strategy, only seven EST-SSR primers were used to distinguish all 22 of the selected grapevine rootstock cultivars in this study. The method is very convenient and fast for the user. Although a single EST-SSR primer cannot distinguish all grapevine rootstock cultivars simultaneously, this method represents a substantial increase in efficiency over previous methods. In addition, it reveals new evidence on the rapid identification of grapevine rootstock cultivars. The informative V-R-CID (Figure 2) of the grapevine rootstock cultivars reveals the specific primers needed to separate specific grapevine rootstock cultivars. Any two or more grapevine rootstock cultivars can be distinguished by the use of one unique primer. For example, the grapevine rootstock cultivars 'shen530' and 'Dogridge' can be distinguished with the use of primer E45 (Figure 3). If the results of PCR amplification show a special band with a fragment size of approximately $220 \mathrm{bp}$, the cultivar can be judged to be 'shen530', otherwise the cultivar is 'Dogridge'. The same principle can be used to distinguish any other two grapevine rootstock cultivars. In practice, if more new grapevine rootstock cultivars are released in production, the set of seven primers selected in this study can be used to run DNA samples of the new cultivars, and the PCR banding patterns can determine the position of the new cultivars in the CID. If they cannot be separated from the 22 cultivars already identified using the seven primers, new primers can be found and used to separate, and then position them on the CID. It appears that not much work needs to be done, and this exercise can generate a larger MCID of grapevine rootstock cultivars, which will definitely prove to be a significant resource for the grapevine rootstock industry. Although the method may not accurately reflect genetic relationships among the cultivars, in theory, the genetic distance between cultivars separated by the first primer is far greater than the distance between cultivars separated by the last primer. This method is a great addition to plant cultivar identification for cultivar-right protection and early identification.

This is the first report on using EST-SSR primers in sequence to identify grapevine rootstock cultivars. In order to verify the reliability of this method, experimental verification, which is an absolute necessity, was conducted and produced satisfactory results. Therefore, this experiment suggests the possibility of utilizing selected DNA markers to distinguish cultivars, even in plant species with a highly heterozygous genome, without requiring a genetic linkage map and/or any DNA sequence information. This technique appears to be effective for the convenient development of selection markers in fruit trees. In addition, these polymorphic bands may be developed into special molecular markers for future cultivar identification. The outstanding results of cultivar identification using this new strategy is that a readable and referable CID can be constructed and used in the classification of related plant species, cultivars, or individual plants in a manner similar to the use of a periodic table of elements in providing the basic information of chemical elements. We believe that as research on this method progresses, this technique and other molecular markers can be used to develop a table for each species, both of plants and other organisms, which in turn can provide us with information 
needed to separate cultivars as desired. There is also a need to test the suitability of different marker types for this method, such as sequence characterized amplified region (SCAR) markers, SSRs, and others, since some markers can be unstable.

In conclusion, this method is rapid, simple, and produces reliable results, since it was possible to demonstrate that a standard set of primers can be used to distinguish a large number of grapevine rootstock cultivars.

\section{ACKNOWLEDGMENTS}

Research supported by grants of the Special Fund for Agro-Scientific Research in the Public Interest (\#201003058-4-2) and the Program of NCET (\#NCET-08-0796).

\section{REFERENCES}

Bassam BJ, Caetano-Anolles G and Gresshoff PM (1991). Fast and sensitive silver staining of DNA in polyacrylamide gels. Anal. Biochem. 196: 80-83.

Bousquet J, Simon L and Lalonde M (1990). DNA amplification from vegetative and sexual tissues of tree using polymerase chain reaction. Can. J. For. Res. 20: 254-257.

Cheng ZP and Huang HW (2009). SSR fingerprinting Chinese peach cultivars and landraces (Prunus persica) and analysis of their genetic relationships. Sci. Hort. 120: 188-193.

D’Onofrio C, De Lorenzis G, Giordani T, Natali L, et al. (2009). Retrotransposon-based molecular markers in grapevine species and cultivars identification and phylogenetic analysis. Acta. Hort. 827: 45-52.

Elidemir AY and Uzun I (2009). Assessment of genetic diversity of some important grape cultivars, rootstocks, and wild grapes in Turkey using RAPD markers. Acta. Hort. 827: 275-278.

Fang J, Qiao Y, Zhang Z, and Chao CT (2005). Genotyping fruiting-mei (Prunus mume Sieb. Et Zucc) cultivars using AFLP. Hort. Sci. 40: 325-328.

Fossati T, Labra M, Castiglione S, Failla O, et al. (2001). The use of AFLP and SSR molecular markers to decipher homonyms and synonyms in grapevine cultivars: the case of the varietal group known as "Schiave". Theor. Appl. Genet. 102: 200-205.

Iezzoni AF and Brettin TS (1998). Utilization of molecular genetics in cherry. Acta. Hort. 498: 53-62.

Lopes MS, Sefc KM, Eiras Dias E, Steinkellner H, et al. (1999). The use of microsatellites for germplasm management in a Portuguese grapevine collection. Theor. Appl. Genet. 99: 733-739.

Lu X, Liu L, Gong Y, Zhao L, et al. (2009). Cultivar identification and genetic diversity analysis of broccoli and its related species with RAPD and ISSR markers. Sci. Hort. 122: 645-648.

Melgarejo P, Martínez JJ, Hernández F, Matínez R, et al. (2009). Cultivar identification using 18S-28S rDNA intergenic spacer-RFLP in pomegranate (Punica granatum L.). Sci. Hort. 120: 500-503.

Murray MG and Thompson WF (1980). Rapid isolation of high molecular weight plant DNA. Nucleic. Acids Res. 8: $4321-4325$.

Papp N, Szilvássy B, Abrankó L, Szabó T, et al. (2010). Main quality attributes and antioxidants in Hungarian sour cherries: identification of genotypes with enhanced functional properties. Int. J. Food. Sci. Technol. 45: 395-402.

Powell W, Morgante M and Andre C (1996). The comparison of RFLP, RAPD, AFLP and SSR (microsatellite) markers for germplasm analysis. Mol. Breed. 2: 225-238.

Reddy PM, Saral N and Siddiq EA (2002). Inter simple sequence repeat (ISSR) polymorphism and its applications in plant breeding. Euphytica 128: 9-17. 